\title{
ESTUDO DA FLUIDODINÂMICA EM LEITO DE JORRO E FLUIDIZADO COM PARTÍCULAS DE SOJA
}

\author{
H. P. FERRAZ ${ }^{1}$, J. H. FERREIRA ${ }^{1}$, F. R. B. D. PORTO ${ }^{1}$ e A. B. OLIVEIRA Jr ${ }^{1}$ \\ ${ }^{1}$ UNIFEB (Centro Universitário da Fundação Educacional de Barretos), Engenharia Química \\ E-mail para contato: fulvi.porto@gmail.com.br
}

\begin{abstract}
RESUMO - Este trabalho teve por objetivo o estudo das curvas características obtidas em leito de jorro e fluidizado com partículas de soja e a comparação dos dados aos modelos fluidodinâmicos presentes na literatura. Foram prédeterminados os modelos: Becker, Malek e Lu. Os procedimentos fluidodinâmicos foram realizados em protótipos de leito jorro e fluidizado, com fluxo ascendente variando-se as velocidades do ar: 0,1 a $20,4 \mathrm{~m} / \mathrm{s}$ e massas: 100 a 500 g. A metodologia mostrou-se adequada tanto na construção dos protótipos como nas coletas de dados, pois possibilitaram reprodutibilidade dos mesmos. Notou-se a influência da altura do leito/massa de partículas na velocidade e observou-se que as curvas obtidas em ambos os leitos foram concordantes com as previstas na literatura por RIBEIRO (2005) e SANTOS (2011).
\end{abstract}

\section{INTRODUÇÃO}

A fluidização é um processo no qual sólidos particulados são colocados em suspensão através de um fluido que pode ser um gás ou um líquido. (TAYLOR e HARMAN, 1990, apud ALMEIDA e ROCHA, p.1).

Segundo GARIM (1998), para o estudo da fluidodinâmica dos leitos é necessário ter-se preliminarmente a sua curva característica (queda de pressão em função da velocidade média do fluido antes do distribuidor) (OLIVEIRA, 2003, p.7).

A curva característica representa fisicamente a transição entre um leito estático e um leito de jorro estabelecido, sendo os trechos da curva ilustrados na Figura 1 (MATHUR e EPSTEIN 1974, apud SANTOS, 2011, p.6). O comportamento de um leito fluidizado apresenta uma curva característica que pode ser observado na Figura 2, sendo descrito por regiões bem definidas. (COUDERC, 1985, apud RIBEIRO, 2005, p.9)

Figura 1 - Curva característica de um leito de jorro

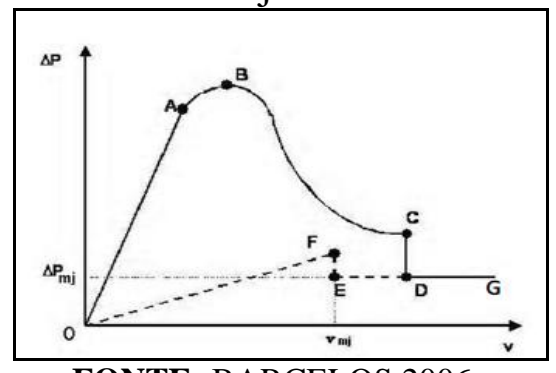

FONTE: BARCELOS 2006, apud SANTOS, 2011
Figura 2 - Curva característica de um leito fluidizado

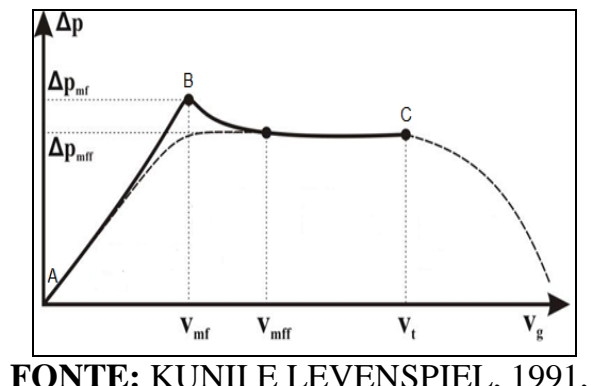
apud MELE, J., 2011 
Para a realização deste estudo foram construídos dois protótipos, sendo eles, um leito de jorro e um leito fluidizado. Utilizou-se como sistema particulado grãos de soja, e os parâmetros analisados foram: velocidade do fluido antes do distribuidor em ambos os leitos e massa de partículas/altura do leito.

\section{MATERIAIS E MÉTODOS}

Para a obtenção das informações experimentais foi utilizado um soprador da marca GE de $127 \mathrm{~V}$, com inversor de frequência para a regulagem da velocidade do fluido, obtida através de um anemômetro ST8020 LCD antes distribuidor posicionado abaixo do leito de partículas. Para cálculo utilizou-se a velocidade média como citado por BRUNETTI (2010), adotando em cada ponto a média das velocidades em relação ao tempo. A perda de carga foi aferida através de um manômetro em U utilizando como fluido manométrico água com corante vermelho, a nivelação do leito foi obtida através de um nível de bolha.

Para construção dos leitos usou-se tubulação de PVC de diversos diâmetros para fração de área livre, junto a estes, acessórios como joelhos, luvas e reduções. Como distribuidor utilizou-se tecido filó antes do início dos leitos. Foi adquirido um funil de calha de zinco com $44,3^{\circ}$ de inclinação e um recipiente cilíndrico de plástico com 14,46 cm de diâmetro e 17,06 cm de altura para o leito de jorro, conforme Figura 3. Para o leito fluidizado foi usado um porta copos de plástico com 7,47 cm de diâmetro e 45,51 cm de altura conforme Figura 4.

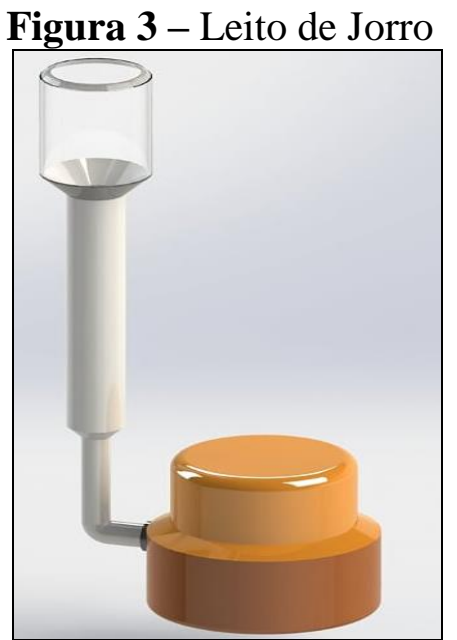

FONTE: Autores
Figura 4 - Leito Fluidizado

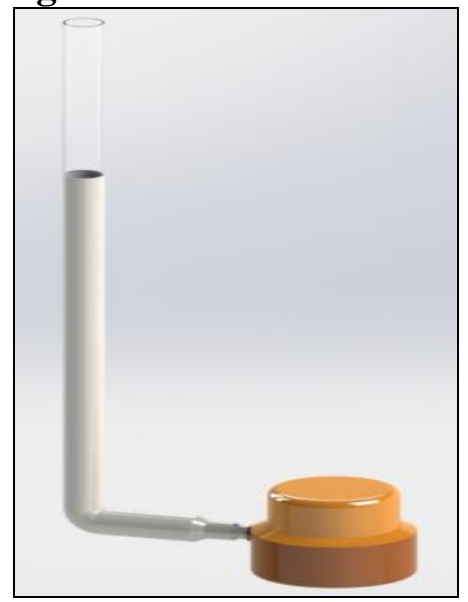

FONTE: Autores

Foram realizados os testes com grãos de soja industrializados (Glycine max), com diâmetro médio de partícula de 5,39 mm, calculado pela Equação 1 descrita por TANNOUS, sendo possível obter a esfericidade de 0,91 através da Equação 2, e o diâmetro equivalente pela Equação 3. Para determinação da densidade aparente, utilizou-se o princípio de Arquimedes para calcular o volume da semente, sendo este pelo deslocamento de água em uma proveta graduada, foram utilizadas 100 sementes, e dividido por sua quantidade para cálculo do volume, como na Equação 4. A porosidade de 0,61 foi obtida pela Equação 5 de CREMASCO (2012), determinando-se a massa do grão utilizando a balança analítica. A densidade real da semente foi calculada através da massa pelo seu volume. E para obtenção da densidade aparente, colocou-se em uma proveta de $250 \mathrm{ml}$ uma quantidade de semente até atingir sua marca, em seguida com a proveta tarada pesou-se para encontrar a massa de 
semente e dividiu pelo volume ocupado na proveta. Todas as coletas foram realizadas em triplicata. A Tabela 1 apresenta as equações utilizadas.

Tabela 1 - Propriedades físicas calculadas

\begin{tabular}{ccc}
\hline Autor & \multicolumn{1}{c}{ Equação } & Numeração \\
\hline Tannous & $d p s=\frac{1}{\sum \frac{x i}{d p i}}$ \\
$d_{p i}=d_{e q} \cdot \emptyset$ & $V_{p=} \frac{\Delta V}{n^{2} d e p a r t i c u l a s}$ \\
Arquimedes & $d_{e q}=\left(\frac{6}{\pi} V_{p}\right)^{\frac{1}{3}}$ \\
Cremasco & $\varepsilon=1-\frac{p_{l}}{p_{p}}$ \\
\hline
\end{tabular}

Para a determinação da fluidodinâmica dos leitos foi elaborado um procedimento operacional padrão de forma a minimizar os erros experimentais e possibilitar a reprodutibilidade das informações experimentais. A queda de pressão foi aferida em função da vazão carregando os leitos com uma massa de grãos/altura do leito, os testes foram realizados variando a vazão de ar nos leitos, sendo esta medida antes do distribuidor.

Para cada vazão obteve-se a diferença de altura no manômetro para o cálculo da perda de pressão através Lei de Stevin (Equação 6). Repetiu-se o teste variando-se a massa do sistema particulado. Através da Equação 7 de PALLAI e NEMETH (1969), obteve-se a máxima queda de pressão teórica possível no leito de jorro, e com a Equação 8 de MALEK e LU (1965), obteve-se a máxima queda de pressão teórica possível no leito fluidizado.

Para se determinar a queda de pressão mínima no leito fluidizado utilizou-se a Equação 10 de BECKER (1961), onde foi necessário determinar porosidade mínima de fluidização (Equação 11), altura do leito na mínima fluidização (Equação 12) e volume total de partículas na mínima fluidização (Equação 13). A queda de pressão mínima em leito de jorro é determinada através da Equação 14, descrita por MASSARANI (1987). A Equação 15 determina o desvio relativo entre a perda de carga teórica e experimental. As equações citadas acima se encontram na Tabela 2.

Tabela 2 - Equações para a determinação da fluidodinâmica dos leitos

\begin{tabular}{|c|c|c|}
\hline Autor & Equação & Numeração \\
\hline Lei de Stevin & $\Delta P=p_{f} \cdot g \cdot h$ & (6) \\
\hline Pallai e Nemeth (1969) & $-\Delta P_{\max }=\left(p_{p}-p_{f}\right)(1-\mathcal{E}) \cdot H_{o} \cdot g$ & (7) \\
\hline \multirow[t]{2}{*}{ Malek e Lu (1965) } & $-\Delta P_{\max }=\frac{g \cdot m_{p}}{\text { Área }}$ & (8) \\
\hline & Área $=\frac{\pi \cdot D^{2}}{4}$ & (9) \\
\hline \multirow[t]{4}{*}{ Becker (1961) } & $\Delta P \min =(1-\mathrm{E} m f) \cdot(\rho p-\rho f) \cdot g \cdot \operatorname{Lm} f$ & $(10)$ \\
\hline & $\emptyset . \mathrm{E} m f=\frac{1}{14}$ & (11) \\
\hline & $V t m f=\frac{\pi \cdot d^{2}}{4} \cdot \operatorname{Lmf}$ & $(12)$ \\
\hline & $V t m f=\frac{V p}{(1-\varepsilon m f)}$ & (13) \\
\hline \multirow[t]{2}{*}{ Massarani (1987) } & $\Delta P \min =0,67 \cdot(1-\varepsilon m f) \cdot$ Ho.p.g & (14) \\
\hline & Desvio relativo $=\frac{|V T-V E|}{V E} .100$ & $(15)$ \\
\hline
\end{tabular}




\section{RESULTADOS E DISCUSSÃO}

As curvas obtidas para o leito de jorro (Figura 5) são semelhantes às curvas apresentadas na literatura (Figura 1), onde no intervalo AB nota-se leito estático, com baixa velocidade do fluido que apenas passa entre as partículas sem as perturbar e a queda de pressão aumenta com a velocidade do gás. Aumentando-se a velocidade do fluido, há uma diminuição gradativa da queda de pressão devido ao aumento do jorro interno e formação do jorro incipiente, como no trecho BC. Prosseguindo com o aumento da velocidade atinge-se o jorro estável, onde a perda de carga se estabiliza como no intervalo CD (MATHUR e EPSTEIN 1974, apud SANTOS, 2011, p.6). Notou-se influência da altura do leito/massa de partículas na velocidade mínima de jorro, esta aumentou juntamente com a altura do leito/massa de partículas assim como a perda de carga.

As curvas obtidas para leito fluidizado (Figura 6) são semelhantes às curvas apresentadas (Figura 2), onde a região $\mathrm{AB}$ corresponde a um leito fixo, e a queda de pressão aumenta à medida que aumenta a velocidade. No ponto B nota-se o começo da fluidização. Com o aumento da velocidade, a queda de pressão estabiliza-se e permanece constante como observado em BC (COUDERC, 1985, apud RIBEIRO, 2005, p.9). Houve influência da altura do leito/massa de partículas na perda de carga, que aumentou proporcionalmente com a altura dos leitos, já na velocidade mínima de fluidização não houve grande influência.

Figura 5 - Curvas características obtidas

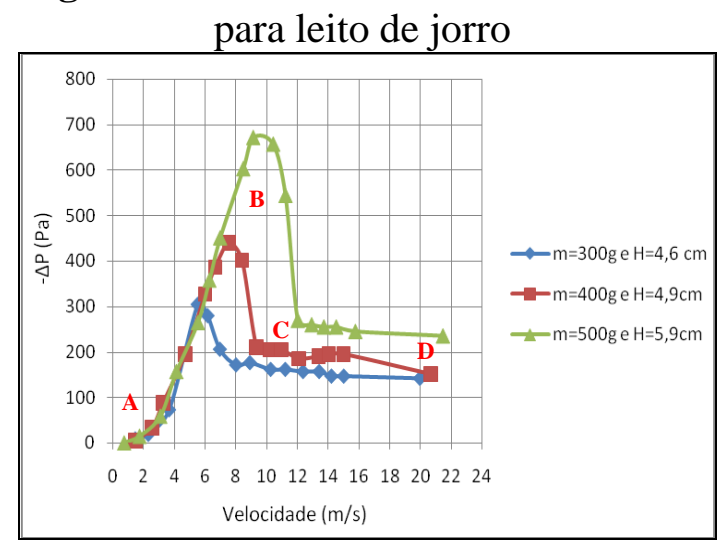

FONTE: Autores
Figura 6 - Curvas características obtidas

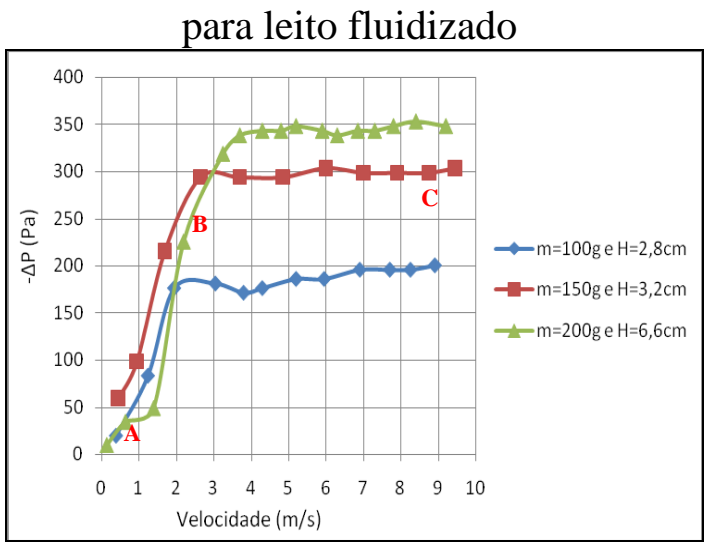

FONTE: Autores

Na Tabela 3 são apresentados os dados experimentais e os teóricos para leito de jorro, juntamente com o desvio relativo obtido, onde a massa especifica do fluido é de $1,179 \mathrm{~kg} / \mathrm{m}^{3} \mathrm{e}$ a massa especifica da particula é de $1742,9 \mathrm{~kg} / \mathrm{m}^{3}$.

Tabela 3 - Dados coletados para leito de jorro

\begin{tabular}{ccccc}
\hline $\boldsymbol{\varepsilon} \mathbf{m f}$ & $\mathbf{H o}(\mathbf{m})$ & $\mathbf{- \Delta} \mathbf{P m i n}$ teórico $(\mathbf{P a})$ & $\mathbf{- \Delta}$ Pmin $\exp (\mathbf{P a})$ & Desvio Relativo (\%) \\
\hline 0,52 & 0,047 & 258,4374 & 235,2 & 9,88 \\
0,52 & 0,045 & 247,4400 & 215,6 & 14,77 \\
0,52 & 0,050 & 274,9334 & 205,8 & 33,60 \\
0,52 & 0,049 & 269,4347 & 215,6 & 24,97 \\
0,52 & 0,060 & 329,9201 & 264,6 & 24,69 \\
0,52 & 0,059 & 324,4214 & 274,4 & 18,23 \\
\hline
\end{tabular}

Os dados experimentas e teóricos para leito fluidizado são apresentados na Tabela 4, juntamente com o desvio relativo obtido. 
Tabela 4 - Dados coletados para leito fluidizado

\begin{tabular}{cccccccc}
\hline $\mathbf{\varepsilon m f}$ & $\mathbf{m p}(\mathbf{k g})$ & $\mathbf{L m f}(\mathbf{m})$ & $\mathbf{V p}\left(\mathbf{m}^{\mathbf{3}}\right)$ & $\mathbf{V t m f}\left(\mathbf{m}^{\mathbf{3}}\right)$ & $\mathbf{- \Delta P m i n}$ teórico (Pa) & $\mathbf{- \Delta P m i n}$ exp (Pa) & Desvio Relativo (\%) \\
\hline 0,52 & 0,10004 & 0,02730 & $5,74 \mathrm{E}-05$ & $1,20 \mathrm{E}-04$ & 223,8917 & 186,2 & 20,24 \\
0,52 & 0,10007 & 0,02731 & $5,74 \mathrm{E}-05$ & $1,20 \mathrm{E}-04$ & 223,9588 & 176,4 & 26,96 \\
0,52 & 0,15008 & 0,04095 & $8,61 \mathrm{E}-05$ & $1,79 \mathrm{E}-04$ & 335,8823 & 294,0 & 14,25 \\
0,52 & 0,15008 & 0,04095 & $8,61 \mathrm{E}-05$ & $1,79 \mathrm{E}-04$ & 335,8823 & 294,0 & 14,25 \\
0,52 & 0,20007 & 0,05460 & $1,15 \mathrm{E}-04$ & $2,39 \mathrm{E}-04$ & 447,7610 & 313,6 & 42,78 \\
0,52 & 0,20015 & 0,05462 & $1,15 \mathrm{E}-04$ & $2,39 \mathrm{E}-04$ & 447,9400 & 323,4 & 38,51 \\
\hline
\end{tabular}

Na comparação entre as máximas quedas de pressão experimentais e calculadas obtevese um desvio relativo médio de $21,02 \%$ para leito de jorro e $26,16 \%$ para leito fluidizado, resultados semelhantes foram obtidos por ALMEIDA, A e ROCHA, S. C. S. (2016). Os desvios relativos devem ser abaixo de $20 \%$, pois estes são satisfatórios de acordo com ALMEIDA, A e ROCHA, S. C. S. Atribui-se os valores superiores ao considerado satisfatório devido a fatores como as condições de contorno das equações utilizadas não serem compatíveis às experimentais, como por exemplo, o ângulo de inclinação do cone do leito de jorro não ser de $60^{\circ}$, não uniformidade das partículas, expansão do leito quando a velocidade se aproxima da velocidade de mínima fluidização e tendência das partículas se agregarem.

\section{CONCLUSÃO}

A metodologia mostrou-se adequada tanto na construção dos protótipos quanto nas coletas de dados, pois possibilitaram reprodutibilidade dos mesmos. Notou-se grande semelhança das curvas características experimentais de ambos os leitos com as presentes na literatura. No estudo fluidodinâmico dos leitos com partículas de soja houve influência da altura do leito/massa de partículas na perda de carga de ambos os leitos, estas aumentaram proporcionalmente com a altura dos leitos, como descrito na literatura. Observou-se influência da altura do leito/massa de partículas na velocidade mínima de jorro, que aumentou juntamente com o aumento da altura do leito/massa de partículas. Não houve grande influência da altura do leito/massa de partículas na velocidade mínima de fluidização.

\section{NOMENCLATURA}

Tabela 5 - Nomenclatura

\begin{tabular}{|c|c|c|c|c|c|}
\hline Símbolo & Descrição & Unidade & Símbolo & Descrição & Unidade \\
\hline D & Diâmetro do leito & $\mathrm{m}$ & $\mathbf{d}_{\mathrm{ea}}$ & Diâmetro equivalente & $\mathrm{m}$ \\
\hline $\mathbf{V}_{\mathrm{mf}}$ & Velocidade mínima de fluidização & $\mathrm{m} / \mathrm{s}$ & $\varnothing$ & Esfericidade & - \\
\hline $\mathbf{H}_{\mathbf{0}}$ & Altura do leito fixo & $\mathrm{m}$ & $\Delta \mathbf{V}$ & Variação de volume & $\mathrm{m}^{3}$ \\
\hline$-\Delta \mathbf{P}$ & Queda de pressão & $\mathrm{Pa}$ & $\varepsilon$ & Porosidade para leito fixo & - \\
\hline d & Diâmetro da partícula & $\mathrm{m}$ & $p f$ & Densidade do fluido & $\mathrm{kg} / \mathrm{m}^{3}$ \\
\hline $\mathbf{V}_{\mathbf{m j}}$ & Velocidade mínima de jorro & $\mathrm{m} / \mathrm{s}$ & $\mathbf{g}$ & Gravidade & $\mathrm{m} / \mathrm{s}^{2}$ \\
\hline$-\Delta \mathbf{P}_{\min }$ & Queda de pressão na min fluidização & $\mathrm{Pa}$ & h & Diferença da altura do líquido & $\mathrm{m}$ \\
\hline$-\Delta \mathbf{P}_{\max }$ & Queda de pressão máxima & $\mathrm{Pa}$ & $\varepsilon_{\mathrm{mf}}$ & Porosidade mínima de fluidização & - \\
\hline$-\Delta \mathbf{P}_{\mathbf{m j}}$ & Queda de pressão no mínimo jorro & $\mathrm{Pa}$ & $\mathbf{m}_{\mathrm{p}}$ & Massa de partículas & $\mathrm{kg}$ \\
\hline$-\Delta \mathbf{P}_{\mathrm{mf}}$ & Queda de pressão na min fluidização & $\mathrm{Pa}$ & Vtmf & Volume de partículas na min. fluidização & $\mathrm{m}^{3}$ \\
\hline$-\Delta \mathbf{P}_{\mathrm{mff}}$ & Queda de pressão na total fluidização & $\mathrm{Pa}$ & Lmf & Altura do leito na mínima fluidização & $\mathrm{m}$ \\
\hline$d_{p i}$ & Diâmetro médio da partícula & $\mathrm{m}$ & $\mathbf{V p}$ & Volume da partícula & $\mathrm{m}^{3}$ \\
\hline$d_{\mathrm{ps}}$ & Diâmetro médio de Sauter & $\mathrm{m}$ & VT & Valor teórico & - \\
\hline $\mathbf{x i}$ & Fração mássica retida & - & VE & Valor experimental & - \\
\hline $\mathbf{V}_{\mathbf{p}}$ & Volume da partícula & $\mathrm{m}^{3}$ & $\mathbf{V}_{\mathrm{t}}$ & Velocidade terminal & $\mathrm{m} / \mathrm{s}$ \\
\hline
\end{tabular}




\section{REFERÊNCIAS}

ALMEIDA, A., ROCHA, S. C. S. Scielo: Fluidodinâmica de semente de brócolis em leito fluidizado e de jorro. Campinas: UNICAMP - Faculdade de Engenharia Química, Universidade Estadual de Campinas, 2001. Artigo (Iniciação científica).

BRUNETTI, F. Mecânica dos fluidos. São Paulo: Pearson Prentice Hall, 2008.

CREMASCO, M. A. Operações unitárias em sistemas particulados e fluidomecânicos. São Paulo: Blucher, 2012.

MARRETO, R. N. Estudo da técnica de leito de jorro convencional para secagem de microcápsulas de caseína e pectina. Ribeirão Preto: USP - Faculdade de Ciências Farmacêuticas de Ribeirão Preto, 2006. Tese (Doutorado).

MELE, J. Cdn: Scale-up of a cold flow model of FICFB biomass gasification process to an industrial pilot plant - example of dynamic similarity. Slovenia: Faculty of mechanical engineering, 2011.

OLIVEIRA, A. B. O. Estudo da transferência de calor e massa no processo de secagem em leito vibro-fluidizado. São Carlos: UFSCAR - Faculdade de Engenharia Química, Universidade Federal de São Calos, 2003. Dissertação (Mestrado).

RIBEIRO, M. S. Estudo fluidodinâmico de um leito fluidizado pulsado rotativo com partículas secas e úmidas. Campinas: UNICAMP - Faculdade de Engenharia Química, Universidade Estadual de Campinas, 2005. Dissertação (Mestrado).

SANTOS, D. A. Contribuições ao estudo da fluidodinâmica em leito de jorro - estudos experimentais e de simulação via CFD. Uberlândia: UFU - Faculdade de Engenharia Química, Universidade Federal de Uberlândia, 2011. Dissertação (Mestrado).

TANNOUS, K., ROCHA, S. C. S. Unicamp: Capítulo III - Escoamento em meios porosos. Disponível em: <https://www.ggte.unicamp.br/ocw/sites/ocw/files/cursos/CienciasExatas/EQ651/apostilas/Capitulo_III.pdf> Acesso 16 set. 2016.

TECNOLOGIA DA FLUIDIZAÇÃO. Tecnologia da fluidização: Leito fluidizado. Disponível em: <http://www.fluidizacao.com.br>. Acesso em 2 set. 2016. 